\title{
EducaÇÃo, Redes Sociais e Sistemas Colaborativos
}

\section{EdUCACIÓN, Redes SOCialeS E SISTEMAS Colaborativos}

Fernanda, Pires $^{1}$, Lilia Valessa, Silva ${ }^{2}$

\author{
${ }^{1}$ Universidade de Lisboa - fernandapires@campus.ul.pt \\ ${ }^{2}$ Universidade Federal do Amazonas - liliaufam@gmail.com
}

Resumo: $O$ presente artigo tem como terceiro objetivo discutir como os espaços de colaboração e de formação são estruturados e materializados nas redes sociais, onde espaços de formação não formais e informais têm como objetivo a popularização da ciência. Os dados e materiais utilizados no trabalho são recuperados da partir da "Fã Page Mundo da Ciência" e do grupo de discussão na mesma página, todos on-line no Facebook.

Palavras Chaves: Educação, Redes Sociais, Espaços Colaborativos

Resumen: El presente articulo tine como objetivo tercer una discursion de cómo se estruturan y se concretan espacios colaborativos y de formación en las redes sociales, donde aparecen espacios de formación no formal e informal objetivando la popularizacíon de la ciência. Para la elaboración del articulo fue utilizado la base de datos y los materiales de la "Fã Page Mundo da Ciência" y del grupo de discurción de la misma página, todas online en el Facebook.

Palabras Clave: Educación, Redes Sociales, Espacios Colaborativos.

Abstract: This article discusses the relationship between cooperation systems and self-organizing systems and how they can be identified in social network. How this spaces can be used to formal and informal training emphasizing how to objective the popularization of science. To this work, was used as database the material of Fan page "Mundo da Ciência" and the group of discussions with the same name, both published in Facebook.

Keywords: Social-Network, Education, Cooperative-systems, 


\section{Introdução}

A integração das TIC's nos varios contextos da sociedade instaurou uma nova era do conhecimento abrindo as portas à utilização da web nos mais diferentes contextos educacionais (Hemmi, Bayne, \& Land, 2009) e as redes sociais não ficaram longe desse processo e permitem uma construção colaborativa de conteúdo. Vivemos o paradigma da globalização, o mundo em rede, conceito observável na world wide web e replicado no conceito de redes sociais que vem demonstrando ter como uma de suas marcas, a cooperação.

Os estudos sobre redes, remontam à primeira metade do século $\mathrm{XX}$, em grupo de mulheres (Davis, Gardner, \& Gardner, 1941), sistemas sociais (Simmel, 1950), relações entre estudantes (Rapoport \& Horvath, 1961,) e mercados de trabalho (Granovetter, 1973). Para Clark (Clark \& Development, 1998)

" network is used to refer to a combination of persons, usually dispersed over a number of geographically separate sites, with appropriate communications technology". (Braga, Gomes, \& Ruediger, 2008) define como rede social "... um grupo de pessoas, de organizações ou de outros relacionamentos, conectados por um conjunto de relações sociais, como as amizades, o trabalho em conjunto ou a simples troca de informações".

Quando estudamos a História da humanidade observamos inúmeras marcas que denotam um comportamento cooperativo, nas relações sociais(Neto, 2000), na indústria (Britto, 2002), na relação entre países(Keohane, 2005).

\section{Enquadramento teórico}

\subsection{A internet e o Facebook}

A internet é um sistema auto-organizado (Palazzo, 2004) e provém meios para que sistemas semelhantes sejam disseminados pela rede, com alguns SNSs (Social Networking Sites). O Facebook é um dos SNSs que reúne o maior número de seguidores. Dados do relatório de 2012 da ITU (Trends in Telecommunication Reform 2012: Smart Regulation for a Broadband World, 2012), apontaram que no mundo todo existem mais um bilhão de usuários de redes sociais, na mesma época o Facebook contava com 901 milhões usuários mensais ativos o que correspondia a 90\% de todas as pessoas ligadas a redes, em 2013 o Facebook conta com 1.19 bilhões de usuários mensais ativos, segundo os dados do Investor Relations ("Facebook Reports Third Quarter 2013 ", 2014).

O Facebook, criado em 2004 tornou-se popular, como uma obsessão social (D. Boyd, 2009; D. E. Boyd, Nicole B, 2007; Lewis, Kaufman, Gonzalez, Wimmer, \& Christakis, 2008; Mayer \& Puller, 2008). Através do Facebook é possível ter um perfil, criar uma rede de contados, gerenciar a prioridade de acesso à sua página pessoal, criar e participar de diferentes grupos, além da possibilidade de curtir fã pages, inscrever-se em páginas para receber atualizações, que variam entre os mais diferentes tipos, piadas, atores, cantores, jornais, organizações internacionais de pesquisa, governo, discussões políticas, etc. e podem ainda participar de grupos, que podem ser públicos, privados, secretos.

Sociólogos, cientistas da informação, físicos e etc. têm entrado nos SNSs e criado redes de conhecimento (Rosenbloom, 2007). Os trabalhos no campo demonstram que as redes tem se expandido e ganhado uma conotação diferente ou sendo integradas em atividades que antes eram realizadas de outra forma. Encontramos na relação entre o SNSs, a produção de conhecimento em redes sociais, sinais que denotam a emergência de sistemas auto-organizados, considerados um grande passo evolutivo no sistema de organização humano (Kauffman, 2005; Moura \& Garcia Pereira, 2003; Palazzo, 2004). A colaboração tem se tornado uma marca nesses SNSs. 


\subsection{Produção Colaborativa}

Para Bruns (2008) a produção colaborativa por pares, em ambientes virtuais, tem aumentado em escala e diversidade em função da produsage, que rompeu muitos dos limites de propriedade privada aplicadas ao conhecimento estabelecido pelos laços de produtores e usuários de conhecimento. A produsage faz com que a figura do emissor e receptor seja obsoleta, pois quem cria é o mesmo que consome e modifica. Isso é possível a qualquer dos agentes conectados a rede, o que aumenta o potencial produtivo e inibe a existência de uma coordenação hierárquica.

$\mathrm{Na}$ busca por um modelo de gestão e/ou ordenação para essa convivência, esses agentes com interesses em comum encontram formas de se articular. Benkler, (2006) apud (d'Andréa, 2012) define que uma das características da produção por pares é emergência de efeitos coordenados, em que o efeito agregador da ação individual, mesmo quando não é conscientemente cooperativo, produz o efeito coordenado de um ambiente informacional novo e rico. Em seguida chama a esse processo de "produção de bens comuns por pares em rede" ressalta que essa ação é "... uma nova modalidade de organizar a produção: radicalmente descentralizada, colaborativa, e não-proprietária; baseada em recursos e produtos amplamente distribuídos, indivíduos conectados de forma flexível que cooperam uns com outros sem se apoiar em informações do mercado ou em comandos gerenciais"

Benkler (2002)citado por d'Andréa (2012) define três características que devem ser marcas na produção por pares: Divisão dos módulos de trabalho em diferentes escalas; independência entre os pares; facilidade de integração entre vários trabalhos produzidos.

O aparecimento de grandes comunidades virtuais, com diferentes níveis de participação, é uma das características da produsage, o sucesso dessa "rede" está diretamente ligado aos três pontos anteriormente estabelecidos. Larsen-Freeman \& Cameron (2008) defendem que a teoria da complexidade tem como objetivo explicar como as partes envolvidas no processo de interação de um sistema complexo originam ao comportamento coletivo do sistema e como esse sistema interage com o ambiente simultaneamente.

\subsection{Sistemas Auto Organizados}

Correia (2006) admite que embora a conceituação de sistemas auto-organizados ainda seja algo impraticável, podemos descrever as propriedades fundamentais da auto-organização e por meio delas definir se um sistema pertence ou não a essa classe. Pelo método da exclusão, se não identificarmos uma dessas características, o sistema não pertence a essa classe.

São características dos sistemas auto-organizados: Inexistência de controle externo, Aumento da ordem (resultante da auto-organização), Adaptabilidade (o sistema deve ser resistente a perturbações, por isso dito robusto), Interação (a existência de vários elementos é mandatória em sistemas autoorganizados. O comportamento resultante do sistema deve ser a implicação das relações entre as partes de acordo com algum critério. Se não fosse dessa forma, os elementos seriam como nós soltos no espaço, sem nenhum comportamento coerente), Assincronismo (inexistência de forma de sincronização global. Em qualquer sistema físico existe um tempo para que o sinal se propague, assim, o mesmo estímulo externo não pode ser percebido por todos em um sistema auto-organizado) (Correia, 2006).

Os trabalhos de (Innes \& Booher, 2000; Johnson, 2001; Moura, 2003; Moura \& Garcia Pereira, 2003) nos dizem que vivemos em uma época de profunda conectividade, a era da informação, de onde emerge uma inteligência coletiva como consequência da interatividade e convergência que a tecnologia digital possibilitou. A interatividade dá origem a stigmergia, a alteração realizada no 
ambiente por um agente age como estímulo a outros agentes e isso dá origem a um comportamento coletivo. "O formigueiro é muito mais inteligente do que cada formiga isolada ou do que a soma da inteligência de todas as formigas" (Moura \& Garcia Pereira, 2003), (Bonabeau, Dorigo, \& Theraulaz, 1999) nomeia como inteligência coletiva ou swarm intelligence.

\subsection{Educação informal e não formal através de redes no Facebook.}

Jonassen (2007) considera a web 2.0 como uma interface social e cognitiva que transforma a maneira como os seres humanos se comunicam, interagem e aprendem. Levy defende que muito pode ser abstraído dessas comunidades, sobretudo ideias e tecnologias cognitivas e que nessa coletividade os groupwares tem uma grande importância (LEVY, 2000; LÉVY, 2000).

O advento da internet foi um catalisador das ações de globalização que já vinham ocorrendo, a formação de grupos ganha outra conotação considerando que as delimitações geográficas não mais são consideradas um problema para a troca de informações. Como defende Pozo (2004), vivemos em uma sociedade da aprendizagem, temos disponíveis várias interfaces e possibilidades de aprender e criar relações em rede.

A UNESCO, (2006) concebe a existência de três tipos de educação, sendo elas: Formal, não formal e informal. Reconhece como educação formal aquela que ocorre no sistema educacional padrão normal; A educação não formal como: aquelas atividades de aprendizagem tipicamente organizadas fora do sistema educacional formal, ou seja, cobre atividades educacionais que visam alfabetização de adultos, educação básica para crianças e jovens que não frequentam a escola. Por fim a educação informal diz respeito a tudo o que acontece cotidianamente na vida dos sujeitos sem objetivos definidos, ou seja, um processo permanente em que cada pessoa adquire atitudes, valores, competências e conhecimentos a partir de sua experiência diária e das influências e recursos educativos de seu entorno, a família, os vizinhos, o trabalho, as atividades recreativas, o mercado, a biblioteca, os meios informativos, etc. (UNESCO, 2008).

Silvestre (2003) vê a educação informal como profundos laços formados ao longo da vida, com ligações intrínsecas com o desenvolvimento pessoal e social, admite ainda que esses laços podem ser perpetuados através de vários meios: Internet, televisão, igreja, família, grupo de amigos da escola, etc. Essa é a educação, ou instrução, que acontece a qualquer momento, nas redes sociais por exemplo, encontramos essas relações que podemos chamar de naturais, entre quem adquire conhecimento e quem disponibiliza as informações para isso. Temos o conceito de produsage, que abrange a disponibilização e criação de conteúdos, propiciando a emergência de redes com interesses em comum, esses ambientes são propícios tanto à educação informal quanto a não formal.

O'reilly (2005) nos diz que para melhor utilizar os espaços disponíveis na web 2.0, é necessário aproveitar o que de melhor ela pode nos proporcionar. No que diz respeito à inteligência coletiva, as melhores ferramentas são aquelas mais eficazes pela maior probabilidade de alcançar o público alvo selecionado. Nesse contexto, isso implica aproveitar os recursos que são utilizados por um amplo número de pessoas pois as possiblidade de impacto são maiores pela abrangência do sinal. Esse comportamento coletivo, é chamado por Alexander (2006) de web social, pois existe a participação colaborativa, o conhecimento é compartilhado com todos, nessas redes e identifica-se uma preocupação com os conteúdos disponibilizados (Alexander, 2006; Bruns, 2007; Garton, Haythornthwaite, \& Wellman, 2006; Pi, Chou, \& Liao, 2013). 


\section{Contexto e objeto de estudo}

O objeto sobre o qual recaiu a análise, é uma comunidade virtual disponível no Facebook, intitulada "Mundo da Ciência", criada em maio de 2013 contando com mais de 130 mil seguidores atéo final de dezembro do mesmo ano. A página foi criada por um grupo que é chamado de formação inicial, composto por 4 pessoas, 3 brasileiros e uma portuguesa, das áreas de Computação, Sociologia, Filosofia e Matemática, o objetivo inicial era compartilhar novas descobertas realizadas no meio científico. Em dezembro de 2013, a comunidade contava com 35 editores, conteúdos cadastrados das mais diferentes áreas do conhecimento.

O objetivo proposto é analisar a dinâmica da comunidade e localizar elementos que nos permitam identificar a presença do padrão inerente aos sistemas auto-organizados em contexto de produsage. Consideramos que as características e conceitos envolvidos nos sistemas complexos e na cooperação podem ajudar na compreensão da dinâmica da produção colaborativa, como a organização social em espaços virtuais pode transpor barreiras criando espaços de educação não formal e informal, inclusive dar suporte a educação formal.

\section{Procedimentos e Instrumentos para recolha de dados:}

Os dados selecionados para análise correspondem aos meses de outubro, novembro e dezembro, a aquisição dos mesmos deu-se por meio de tabelas em formato XML disponibilizadas pela plataforma administrativa do Facebook aos administradores da página, importação de dados via módulo social do NodeXL, e pelo aplicativo Netvizz. As postagens são realizadas pelos membros administradores e as discussões que seguem são realizadas pelos comentadores da página, sem nenhuma estrutura hierárquica, nem mesmo entre o grupo de moderadores.

Agem como pares,trocam informações, reconstroem o conhecimento, adicionam novas informações a mensagem. A dinâmica da página pode ser definida por seu alcance, mensurável pelo número de posts, número de comentários por cada post, número de pessoas que passam a seguir a página, número de pessoas que deixam de seguir a página.

Tabela 1: Alcance da MdC nos três últimos meses de 2013

\begin{tabular}{|l|l|l|l|l|l|}
\hline Meses & Total de Curtidas & Novatos & Saídas & Media Interação & № posts \\
\hline Outubro & 99175 & 4514 & 540 & 4.581 & 445 \\
\hline Novembro & 117549 & 17867 & 1161 & 22.521 & 473 \\
\hline Dezembro & 131050 & 14464 & 923 & 22.925 & 479 \\
\hline
\end{tabular}

Fonte: elaboração dos autores

A tabela mostra que existe um crescimento evidente, e que embora exista um número de pessoas que deixam curtir a página (saídas), a aderência é bem maior. Localizamos uma dinâmica de formação de redes pela relação entre os eventos, através do compartilhamento da informação e das interações advindas de cada um dos posts, criando uma rede dinâmica de compartilhamento, nem todos os likes advém dos seguidores cadastrados na página, ou seja, o alcance vai para além das "assinaturas" mensais.

Observamos também um equilíbrio não proposital entre o número de posts por mês, apesar não haver nenhuma regra clara entre os gerenciadores de conteúdo, existe um equilíbrio entre o número de postagens mensais, com uma margem não superior a $8 \%$, como demonstram a tabela e o gráfico a seguir: 


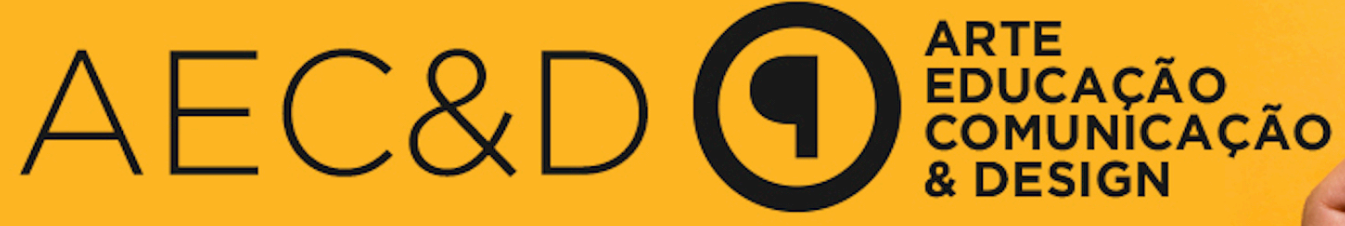

Tabela 2: Posts Mdc 2013

\begin{tabular}{|l|l|l|l|l|}
\hline Meses & Postagens & Comentários & "Like" & Share \\
\hline Outubro & 445 & 5396 & 49265 & 17900 \\
\hline Novembro & 473 & 18363 & 134972 & 43382 \\
\hline Dezembro & 479 & 22881 & 179473 & 56307 \\
\hline
\end{tabular}

Fonte: elaboração dos autores

Notamos uma diferença entre o mês de outubro e os demais, se considerarmos uma descida no sistema, é observável que houve uma recuperação em seguida.Como reflexo da interação na rede, a comunidade Mundo da Ciência se expandiu através da criação de grupos menores, é previsível maior interação entre os membros por meio de discussões.

O grupo de debates da MdC é um domínio fechado, ou seja, precisa de convites, mesmo assim observamos a emergência de grupos, redes dentro de redes, muitos vértices ligados aos administradores. O grau do nó foi definido pelo número de interações, essa participação é recompensada por meio de aquisição de privilégios, por exemplo, o indivíduo com grande número de participações, ganhou o status de administrador do grupo, que são representados pelos maiores nós. (Figura 01).

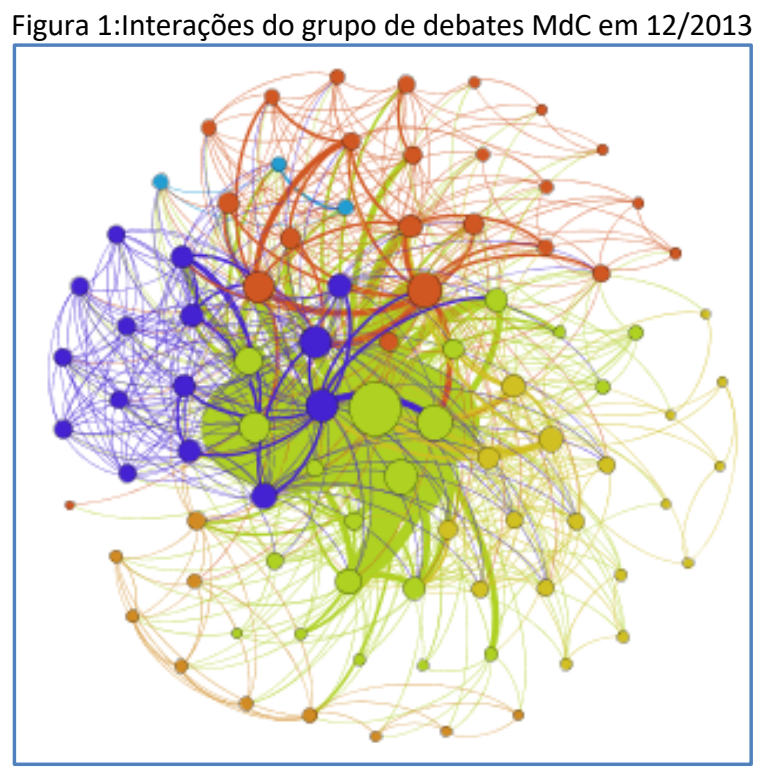

Fonte: elaboração dos autores

A cooperação sem dúvida é uma marca do grupo, o crescimento do alcance dá-se graças à colaboração de filiados e não filiados que fazem com que os posts se estendam na rede e ganhem maior visibilidade. É uma rede dinâmica, pois a cada dia ganha novos adeptos, sendo impossível prever o seu comportamento diante do próximo quadro. A extração de dados referente a 600 postagens, resultou no gráfico que se demontra abaixo. Nesse gráfico é possivel obervar a formação de 29 comunidades que emergem a partir das 600 postagens na página da comunidade MdC. No intervalo avaliado, classificamos a rede como dinâmica, segundo os padrões (Barabâsi et al., 2002), a formação deu-se por meio de pontos de interesse em comum entre os temas publicados. 


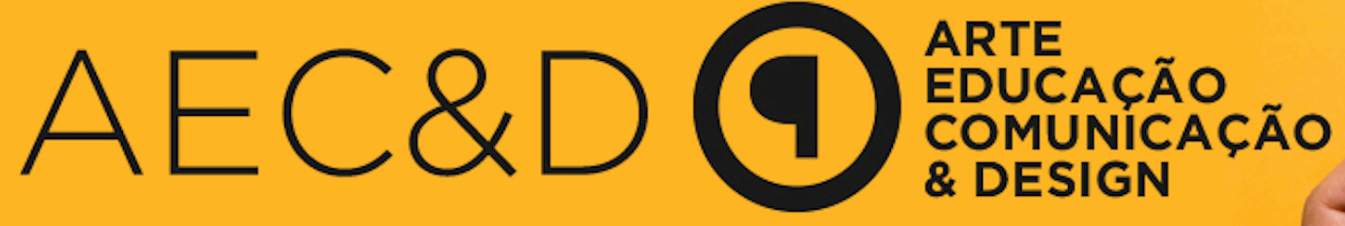

Figura 2:Fomação de comunidades em torno dos tópicos

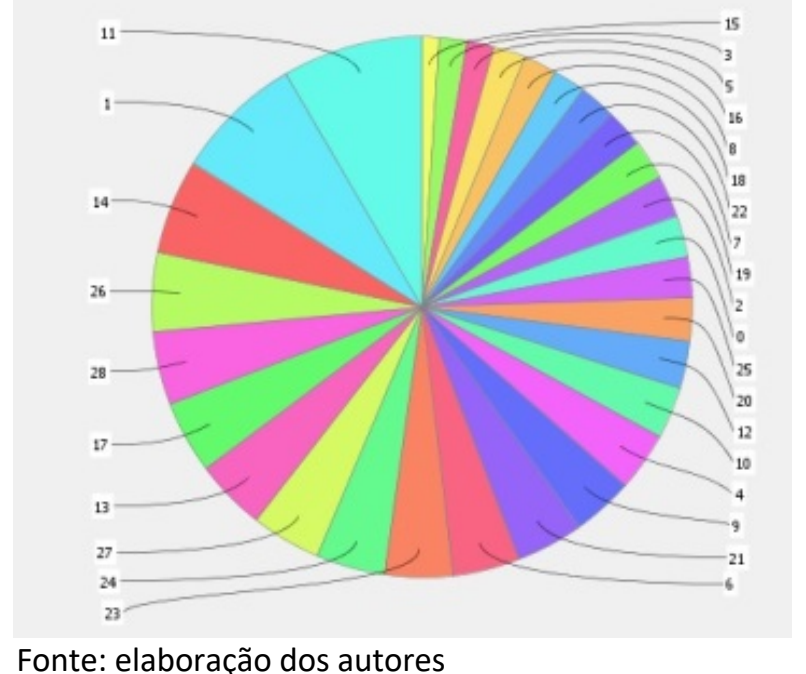

Fonte: elaboração dos autores

\section{Resultados e Discussão}

O estudo sobre redes e cooperação em sociedades do conhecimento é um terreno em expansão que pode auxiliar na educação formal e informal (Liu, Jiang, \& Ma, 2013; Martins; Martins \& Ferreira, 2013; Scott, 2004; Shin \& Kook; Traud, Mucha, \& Porter, 2012). Quanto as característica dos sistemas autoorganizados discutidos anteriormente, notou-se que (Tabela 3):

Tabela 3 - Característica dos sistemas auto-organizados

\begin{tabular}{|l|l|}
\hline Inexistência de controle externo & MdC \\
\hline Aumento da ordem & $\begin{array}{l}\text { Apesar de contar com gerentes de conteúdo, estes não tem como controlar uma } \\
\text { comunidade tão grande. Inexistência de hierarquia, o comportamento coletivo de } \\
\text { agentes simples cria um padrão de comunicação. A influencia desses agentes no } \\
\text { meio faz com que se crie um padrão coletivo que pode dar respostas a problemas } \\
\text { maiores, essa troca de informações, que dão origem a padrões, podemos chamar } \\
\text { de stigmergia. }\end{array}$ \\
\hline Adaptabilidade & $\begin{array}{l}\text { O sistema mantém certa ordem pela estabilidade encontrada no número de posts } \\
\text { mensais, independente do número de gerenciadores de conteúdo ativos. Outubro } \\
\text { contou com 11 gerentes ativos, novembro contou com 10 e dezembro com 14, } \\
\text { embora a comunidade conte com 40 membros cadastrados como produtores de } \\
\text { conteúdo. }\end{array}$ \\
\hline Interação & $\begin{array}{l}\text { O sistema tem se mostrado resistente a perturbações, como as saídas ou pessoas } \\
\text { insatisfeitas com o material publicado. }\end{array}$ \\
\hline Assincronia & $\begin{array}{l}\text { Os números referentes a comentários, adesões, "likes" e compartilhamentos, além } \\
\text { do surgimento de grupos menores em torno de temas discutidos pela comunidade, } \\
\text { tem mostrado um aumento crescente na interatividade. }\end{array}$ \\
\hline $\begin{array}{l}\text { Um post não chega a toda a comunidade ao mesmo tempo, as pessoas podem ou } \\
\text { não receber notificações de objetos de interesse. Não existe uma ordem de } \\
\text { postagem estabelecida, a propagação é relativa e depende dos nós que receberão o } \\
\text { estímulo. }\end{array}$ \\
\hline
\end{tabular}

Fonte: elaboração dos autores

Em se tratando de processos educacionais, pode a formação de redes de conhecimento não formal e informal auxiliar o processo de educação formal ou ainda ser um suporte permanente a educação não formal? Segundo os dados observacionais e numéricos coletados, pode-se afirmar que é inegável que a publicitação de informações de cunho científico e social não afete os constructos das pessoas que a partir de discussões, criam novos constructos. 
A MdC é um ambiente cujos conteúdos estimulam a pesquisa, a interação, formação de grupos de discussão, grupos de estudo, compartilhamento de experiências, formação de redes de conhecimento afins, a multidisciplinaridade, a interdisciplinaridade, entre outras características essenciais ao desenvolvimento de programas com fundo educacionais.

Os SNSs podem prove uma nova ferramenta para os processos educativos, seja no campo da construção conjunta, estimulação a cooperatividade, currículo educacional mais amplo ou até mesmo uma proposta de ensino via pesquisa. Os SNSs são amplamente utilizados em dispositivos móveis, sendo mais uma forma de computação ubíqua, onde a tecnologia vai até o seu usuário e não o contrário.

Segundo os dados que obtivemos, consideramos que pelo menos $90 \%$ das pessoas interagiram com a página nos três meses analisados, utilizou como plataforma de educação informal, pois pelo menos uma vez compartilhou, curtiu ou comentou. Comentários no grupo de debates evidenciam a criação de grupos com interações mais fortes, prova disso é o plano de criação de um website para tirar dúvidas sobre as mais diferentes áreas do conhecimento. A gestão será realizada pelos membros interessados em produzir conteúdo, não existe nenhuma forma de retribuição financeira, a "moeda corrente", nesse ambiente, é o reconhecimento pelo trabalho realizado. $\mathrm{O}$ alcance dos posts se mostra um bom estímulo aos produtores de conteúdo.

\section{Considerações finais}

O SNSs podem tornar-se importantes aliados no processo de aquisição de competências. Estudos comprovam que as redes tem obtido cada vez mais adeptos e pode ser um espaço de compartilhamento de informações que podem ou não ter conteúdos direcionados. A identificação de características de sistemas auto organizados e inteligência de enxame nessas comunidades pode ser muito útil no planejamento de ações futuras no campo educacionalObservou-se que além da natureza onde encontramos os sistemas auto-organizados frequentemente, podemos observar o mesmo padrão em várias novas "invenções", como a internet, as redes de computadores, as redes sociais digitais, coisas que há algum tempo seriam impensáveis. É interessante a possibilidade de aplicarmos conceitos iguais a diferentes áreas do conhecimento, a multidisciplinaridade implicada nesse fator. Seria essa mais uma forma de nos tornarmos permeáveis as mudanças, a diversidade e criar cada vez mais mecanismos evolucionários?

Browloo e Smith, (Brownlee; 2000) defendem que o processo de aprendizagem interativa, realizada por grupos, cria vários complexos de capacidades tecnológicas e determina certa dinâmica do sistema do qual podem emergir padrões de inovação. A comunidade Mdc tem mostrado a possibilidade dessa afirmação através do surgimento de interesses a partir de postagens que desencadeiam eventos como reportagens em série e discussões politicas sobre temas como sedimentação do solo e como isso é tratado nas diversas regiões.

\section{Referencias}

Alexander, B. (2006). Web 2.0: A new wave of innovation for teaching and learning? Educause review, 41(2), 32.

Barabâsi, A.-L., Jeong, H., Néda, Z., Ravasz, E., Schubert, A., \& Vicsek, T. (2002). Evolution of the social network of scientific collaborations. Physica A: Statistical Mechanics and its Applications, 311(3), 590614.

Benkler, Y. (2002). Coase's Penguin, or, Linux and" The Nature of the Firm". Yale Law Journal, 369-446. Benkler, Y. (2006). The wealth of networks: How social production transforms markets and freedom: Yale University Press. 
Bonabeau, E., Dorigo, M., \& Theraulaz, G. (1999). Swarm intelligence: from natural to artificial systems (Vol. 4): Oxford university press New York.

Boyd, D. (2009). Why youth (heart) social network sites: The role of networked publics in teenage social life.

Boyd, D. E., Nicole B. (2007). Social network sites: Definition, history, and scholarship. Journal of Computer-Mediated Communication, 13(1), 210-230.

Braga, M. J. d. C., Gomes, L. F. A. M., \& Ruediger, M. A. (2008). Mundos pequenos, produção acadêmica e grafos de colaboração: um estudo de caso dos Enanpads. rap-rio de Janeiro, 42(1), 133-154.

Britto, J. (2002). Cooperação interindustrial e redes de empresas. Economia industrial: fundamentos teóricos e práticos no Brasil. Rio de Janeiro: Campus, 345-388.

Brownlee, J. Unexplored Territory: Seeds For Future Research Investigations.

Bruns, A. (2007). Produsage. Paper presented at the Proceedings of the 6th ACM SIGCHI conference on Creativity \& cognition.

Bruns, A. (2008). Blogs, Wikipedia, Second Life, and beyond: From production to produsage (Vol. 45): Peter Lang.

Clark, H. C., \& Development, I. I. f. S. (1998). Formal Knowledge Networks [electronic Resource]: a Study of Canadian Experiences: International Institute for Sustainable Development= Institut international du développement durable.

Correia, L. (2006). Self-organised systems: fundamental properties Revista de Ciências da Computação, I(I), 1-10.

d'Andréa, C. F. d. B. (2012). Wikipédia como um sistema adaptativo complexo: auto-organização e emergência na produção por pares//WIKIPEDIA AS AN COMPLEX ADAPTIVE SYSTEM: SELFORGANIZATION AND EMERGENCE IN PEER PRODUCTION. Contemporanea-Revista de Comunicação e Cultura, 10(3), 490-510.

Davis, A., Gardner, B. B., \& Gardner, M. R. (1941). Deep south: University of Chicago Press Chicago.

Dugatkin, L. A., \& Reeve, H. K. (1997). Cooperation among animals: an evolutionary perspective: Oxford University Press Oxford.

Facebook Reports Third Quarter 2013 (2014, 10/01/2013). Retrieved 12/01/14, 2014, from http://investor.fb.com/releasedetail.cfm?ReleaselD $=802760$

Garton, L., Haythornthwaite, C., \& Wellman, B. (2006). Studying online social networks. Journal of Computer-Mediated Communication, 3(1), 0-0.

Granovetter, M. S. (1973). The strength of weak ties. American journal of sociology, 1360-1380.

Hemmi, A., Bayne, S., \& Land, R. (2009). The appropriation and repurposing of social technologies in higher education. Journal of Computer Assisted Learning, 25(1), 19-30.

Innes, J. E., \& Booher, D. E. (2000). Network power in collaborative planning.

Johnson, S. (2001). Emergence: The Connected Lives of Ants, Brains, Cities, and Software.

Jonassen, D. H. (2007). Computadores, Ferramentas Cognitivas-Desenvolver o pensamento crítico nas escolas. Porto: Porto Editora.

Kauffman, S. (2005). O universo, a nossa casa. A procura das leis da auto.

Keohane, R. O. (2005). After hegemony: Cooperation and discord in the world political economy: Princeton University Press.

Kirkpatrick, D. (2011). The Facebook effect: The inside story of the company that is connecting the world: Simon \& Schuster.

Larsen-Freeman, D., \& Cameron, L. (2008). Complex systems and applied linguistics: Oxford University Press.

LEVY, P. (2000). A inteligência Coletiva-Por uma antropologia do ciberespaço, Loyola: São Paulo.

Lévy, P. (1999). Cibercultura: Editora 34-FRETE GRATIS!

LÉVY, P. (2000). As tecnologias da inteligência: o futuro do pensamento na era da informática. Rio de Janeiro: Editora 34, 1993 (Coleção TRANS). _. Cibercultura. São Paulo: Editora, 34.

Lewis, K., Kaufman, J., Gonzalez, M., Wimmer, A., \& Christakis, N. (2008). Tastes, ties, and time: A new social network dataset using Facebook. com. Social networks, 30(4), 330-342. 
Liu, X., Jiang, T., \& Ma, F. (2013). Collective dynamics in knowledge networks: Emerging trends analysis. Journal of Informetrics, 7(2), 425-438. doi: http://dx.doi.org/10.1016/j.joi.2013.01.003

Martins, D. L. Identificação de Presença de Grupos em Grafos por Níveis de Distribuição de Grau de Centralidade.

Martins, D. L., \& Ferreira, S. M. S. P. (2013). Dinâmica de constituição e eventos geradores em redes de colaboração científica: estudo exploratório entre relações de coautoria e em bancas de defesas de teses e dissertações. Em Questão, 18(3).

Mayer, A., \& Puller, S. L. (2008). The old boy (and girl) network: Social network formation on university campuses. Journal of public economics, 92(1), 329-347.

Moura, L. (2003). Formigas, vagabundos e anarquia: ensaio sobre vida artificial arte e sociedade.

Moura, L., \& Garcia Pereira, H. (2003). Aprendendo com a stigmergia, a auto-organização e as redes de cooperação. Paper presented at the III Conferência Internacional sobre Tecnologias de Informação e Comunicação na Educação.

Neto, J. A. (2000). Redes de cooperação produtiva e clusters regionais: oportunidades para as pequenas e médias empresas: Editora Atlas.

O'reilly, T. (2005). What is web 2.0.

Palazzo, L. A. M. (2004). Complexidade, caos e auto-organização. Retrieved October, 4, 2006.

Pi, S.-M., Chou, C.-H., \& Liao, H.-L. (2013). A study of Facebook Groups members' knowledge sharing. Computers in Human Behavior, 29(5), 1971-1979. doi: http://dx.doi.org/10.1016/j.chb.2013.04.019

Pozo, J. I. (2004). A sociedade da aprendizagem eo desafio de converter informação em conhecimento. Revista Pátio, Porto Alegre, ano VIII(31).

Rapoport, A., \& Horvath, W. J. (1961). A study of a large sociogram. Behavioral Science, 6(4), 279-291. Rosenbloom, S. (2007). On Facebook, scholars link up with data. New York Times, 17.

Scott, J. (2004). Social network analysis: a handbookSocial network analysis: a handbook www.analytictech.com/mb119/tableof.htm.

Shin, S. K., \& Kook, W. Can Knowledge Be More Accessible in a Virtual Network?: Collective Dynamics of Knowledge Transfer in a Virtual Knowledge Organizations Network. Decision Support Systems(0). doi: http://dx.doi.org/10.1016/j.dss.2013.11.006

Silvestre, A. C. (2003). Educação/Formação de Adultos. Porto Alegre: Instituto Piaget.

Simmel, G. (1950). The sociology of georg simmel (Vol. 92892): SimonandSchuster. com.

Smith, K. (2000). Innovation as a systemic phenomenon: rethinking the role of policy. Enterprise and innovation management studies, 1(1), 73-102.

Traud, A. L., Mucha, P. J., \& Porter, M. A. (2012). Social structure of Facebook networks. Physica A: Statistical Mechanics and its Applications, 391(16), 4165-4180. doi: http://dx.doi.org/10.1016/j.physa.2011.12.021

Trends in Telecommunication Reform 2012: Smart Regulation for a Broadband World. (2012). International Telecommunication Union.

UNESCO. (2008). Informe de seguimiento de la EPT en el mundo. Bases sólidas: atención y educación de la primera infância. Paris: UNESCO.

UNESCO

Burnett, N. (2006). La alfabetización un factor vital. Informe de seguimiento de la EPT en el mundo. Paris: Paris: UNESCO.

Zheng, R., Wilkinson, D., \& Provost, F. (2008). Social network collaborative filtering. Stern, IOMS Department, CeDER, Vol. 